03

\title{
Изменение свойств турбулентных течений в сферическом слое под действием модуляции скорости вращения
}

\author{
() Д.Ю. Жиленко, О.Э. Кривоносова
}

Институт механики МГУ им. М.В. Ломоносова, Москва

E-mail: jilenko@imec.msu.ru

Поступило в Редакцию 10 ноября 2016 г.

Экспериментально исследованы изотермические турбулентные течения вязкой несжимаемой жидкости в сферическом слое. Рассматривается воздействие модуляции скорости вращения внутренней сферы на свойства турбулентности. Проведены измерения скорости течения лазерным доплеровским анемометром. Обнаружен локальный минимум в зависимости величины турбулентных пульсаций от амплитуды модуляции. Установлено, что в отсутствие модуляции наклоны спектров скорости близки к величине $-11 / 5$. С увеличением амплитуды модуляции возможно формирование спектров, характерных для двумерной турбулентности: с наклоном $-5 / 3$ на низких частотах и наклоном -3 на высоких частотах.

DOI: $10.21883 /$ PJTF.2017.10.44625.16564

Основным свойством турбулентных течений является наличие инерционного интервала переноса энергии [1]. Взаимное расположение участков энергетического спектра и их наклон в инерционном интервале определяют вид турбулентности. В трехмерной турбулентности наблюдается прямой каскад переноса энергии от больших к меньшим масштабам [1-3]. Зависимость энергетического спектра $E(k)$ от волнового числа $k$ в трехмерной турбулентности имеет вид $E(k) \sim k^{-5 / 3}$ для изотермических течений и $E(k) \sim k^{-11 / 5}$ для стратифицированных по температуре течений [1]. В двумерной турбулентности при меньших частотах наблюдается обратный каскад передачи кинетической энергии от меньших масштабов к большим с зависимостью $E(k) \sim k^{-5 / 3}$, а при больших частотах - прямой каскад переноса энстрофии с зависимостью $E(k) \sim k^{-3}[2,3]$. Двумерная и трехмерная турбулентность могут наблюдаться одновременно в различных пространственных областях 
одного и того же течения [4,5]. Переход от трехмерной к двумерной турбулентности в течении может происходить при наличии внешней вынуждающей силы и вращения [6,7]. Сочетание вращения и сферической геометрии, характерное для крупномасштабных геофизических процессов, учитывается в сферическом течении Куэтта (СТК) $[8,9]$ - течении вязкой несжимаемой жидкости, вызванном вращением концентрически расположенных сферических границ вокруг общей оси. В [9] показано, что в результате ламинарно-турбулентного перехода, вызванного периодической модуляцией скорости вращения одной из границ, в СТК могут формироваться и двумерная, и трехмерная турбулентность. Выводы о виде турбулентности в [9] получены как по наклону спектров, так и по знаку структурной функции третьего порядка скорости [1]. Тем не менее остается открытым вопрос о восприимчивости развитой турбулентности, формирующейся в СТК при постоянных скоростях вращения границ, к воздействию модуляции. Несмотря на успехи моделирования турбулентности различными способами $[5,6,10]$, неединственность решений уравнений Навье-Стокса определяет необходимость в том числе и экспериментальных исследований при изучении турбулентности.

Цель настоящей работы - экспериментальное исследование влияния модуляции скорости вращения внутренней сферы на свойства развитых турбулентных течений во вращающемся сферическом слое.

Эксперименты проводились в сферическом слое, сформированном двумя оптически прозрачными коаксиальными сферами, с радиусами внутренней и внешней сфер $r_{1}=0.075$ и $r_{2}=0.15 \mathrm{~m}$ соответственно. Слой наполнялся силиконовым маслом с вязкостью $v \approx 5 \cdot 10^{-5} \mathrm{~m}^{2} / \mathrm{s}$ при температуре $22^{\circ} \mathrm{C}$. Для стабилизации температуры сферы размещены в заполненном силиконовым маслом термостате. Температура рабочей жидкости в слое поддерживалась постоянной, с точностью не менее $\pm 0.05^{\circ} \mathrm{C}$, и контролировалась расположенным на экваторе внешней сферы датчиком. Измерения азимутальной компоненты скорости течения $u_{\varphi}$ лазерным анемометром проводились вблизи внешней сферы. Точка измерения удалена от плоскости экватора на расстояние $0.078 \mathrm{~m}$, и от оси вращения на $0.105 \mathrm{~m}$. Скорость вращения внешней сферы $\Omega_{20}$ поддерживалась постоянной. Скорость вращения внутренней сферы $\Omega_{1}(t)$ изменялась с задаваемой системой управления амплитудой $A$ и частотой $f$ со средней погрешностью не более $0.05 \%$ от заданных значений: $\Omega_{1}(t)=\Omega_{10}(1+A \sin (2 \pi f t+\varphi))$. Величины $f=0.01$ и $0.08 \mathrm{~Hz}$ не превышали средних величин частот 


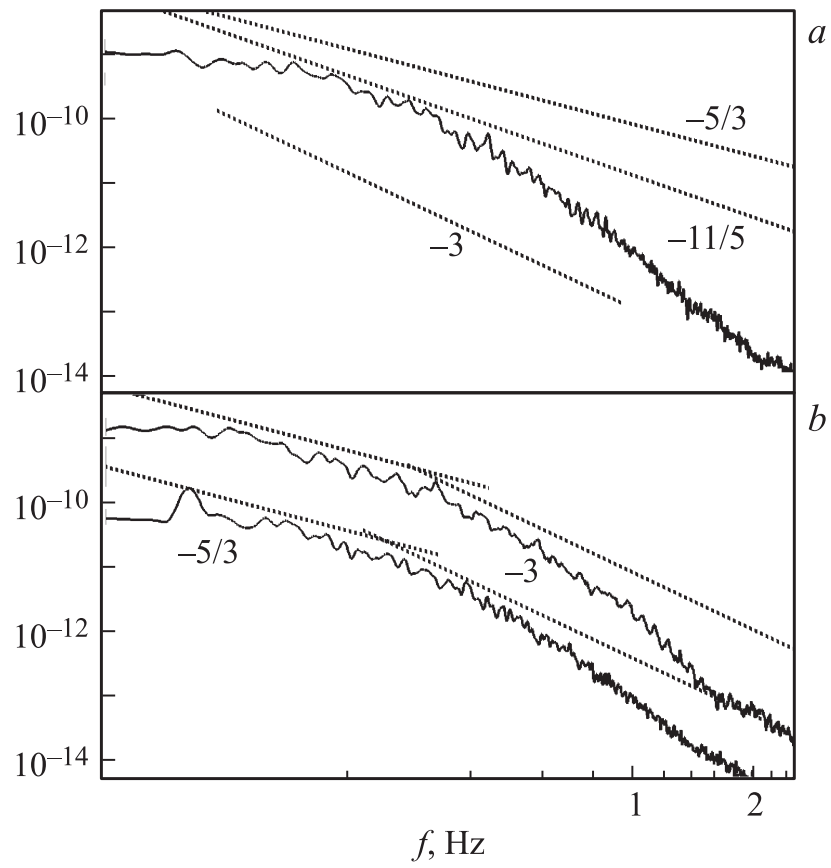

Рис. 1. Полученные в эксперименте спектры $u_{\varphi}^{2}: a-A=0 ; b-f=0.01 \mathrm{~Hz}$, $A=0.078$ (верхняя кривая), $f=0.08 \mathrm{~Hz}, A=0.1165$ (нижняя кривая, для удобства восприятия сдвинута вниз на 2 порядка по оси ординат).

вращения сфер. Эксперименты проводились следующим образом: сначала при встречном вращении границ и $A=0$ формировалось исходное турбулентное течение при числах Рейнольдса $\mathrm{Re}_{1}=\Omega_{10} r_{1}^{2} / v=450$ и $\operatorname{Re}_{2}=\Omega_{20} r_{2}^{2} / v=-900$. Далее при выбранной величине $f$ амплитуду $A$ увеличивали от 0 до заданного значения (но не более $12 \%$ от $\Omega_{10}$ ).

Обработка результатов измерений показала, что в отсутствие модуляции в спектре можно выделить участок с постоянным углом наклона, близким к величине $-11 / 5$ (рис. 1,a). Выше отмечалось, что зависимость вида $E(k) \sim k^{-11 / 5}$ характерна для турбулентности в стратифицированных по температуре течениях [1], и ранее подобные зависимости экспериментально наблюдались только при исследовании

Письма в ЖТФ, 2017, том 43, вып. 10 
Области формирования различных спектров турбулентности в зависимости от амплитуды $A$ и частоты $f$ модуляции (темным цветом показаны реализуемые в эксперименте спектры)




конвекции (например, в [11]). В нашем случае контролируемые величины температур рабочей жидкости в слое и термостате исключают появление конвекции. Необходимо отметить, что общим свойством рассматриваемого турбулентного течения и турбулентных конвективных течений является наличие крупномасштабной циркуляции и, возможно, именно это является причиной формирования турбулентных спектров одного и того же вида. При $A \neq 0$ вид спектров зависит от $f$. В случае $f=0.01 \mathrm{~Hz}$ при $A<4 \%$ рассматриваемый выше спектр с наклоном $-11 / 5$ сохраняется (см. таблицу). Дальнейшее повышение $A$ ведет к формированию турбулентных спектров с наклоном $-5 / 3$ (рис. $1, b$ ), характерных для трехмерной турбулентности в изотермических течениях (см. таблицу). Такие же спектры формируются в случае $f=0.08 \mathrm{~Hz}$ при $A<10 \%$ (см. таблицу). Дальнейшее увеличение амплитуды приводит к появлению спектров, характерных для двумерной турбулентности: c наклоном $-5 / 3$ на меньших частотах и наклоном -3 на больших частотах (рис. $1, b)$. Граница между интервалами с различным наклоном спектра соответствует средней частоте вращения внутренней сферы.

Рассмотрим, каким образом увеличение $A$ и $f$ воздействует на вид спектров. Сначала наклон спектра снижается на низких частотах: на рис. 1 виден переход от наклона $-11 / 5$ к наклону $-5 / 3$ в одном и том же диапазоне частот. Затем на частотах больших, чем частота вращения внутренней сферы, наклон спектра возрастает до величины -3 (рис. $1, b$ ). Такое изменение спектров свидетельствует о повышении энергии турбулентности на высоких частотах с увеличением амплитуды модуляции. Изменение вида спектров является следствием изменения свойств турбулентных пульсаций скорости. Так, изображенные на рис. 2, $a$ зависимости среднеквадратичных отклонений $u_{\varphi} \operatorname{rms} u_{\varphi}=\sqrt{\frac{1}{N_{t}} \sum_{n=1}^{N_{t}}\left(u_{\varphi}(n, r)-\overline{u_{\varphi}(r)}\right)^{2}}$ от $A$ показывают наличие локальных минимумов при $A<0.05$, и чем меньше величина $f$, тем более выражен этот минимум. Таким образом, при небольших амплитудах модуляции возможно уменьшение уровня турбулентных пульсаций по сравнению со случаем $A=0$. В том диапазоне изменения $A$, где происходит переход от трехмерной турбулентности к двумерной в случае $f=0.08 \mathrm{~Hz}$ (см. таблицу), наблюдаются наибольшие отличия в уровне турбулентных пульсаций: для двумерной турбулентности они существенно ниже, чем для трехмерной.

Письма в ЖТФ, 2017, том 43, вып. 10 


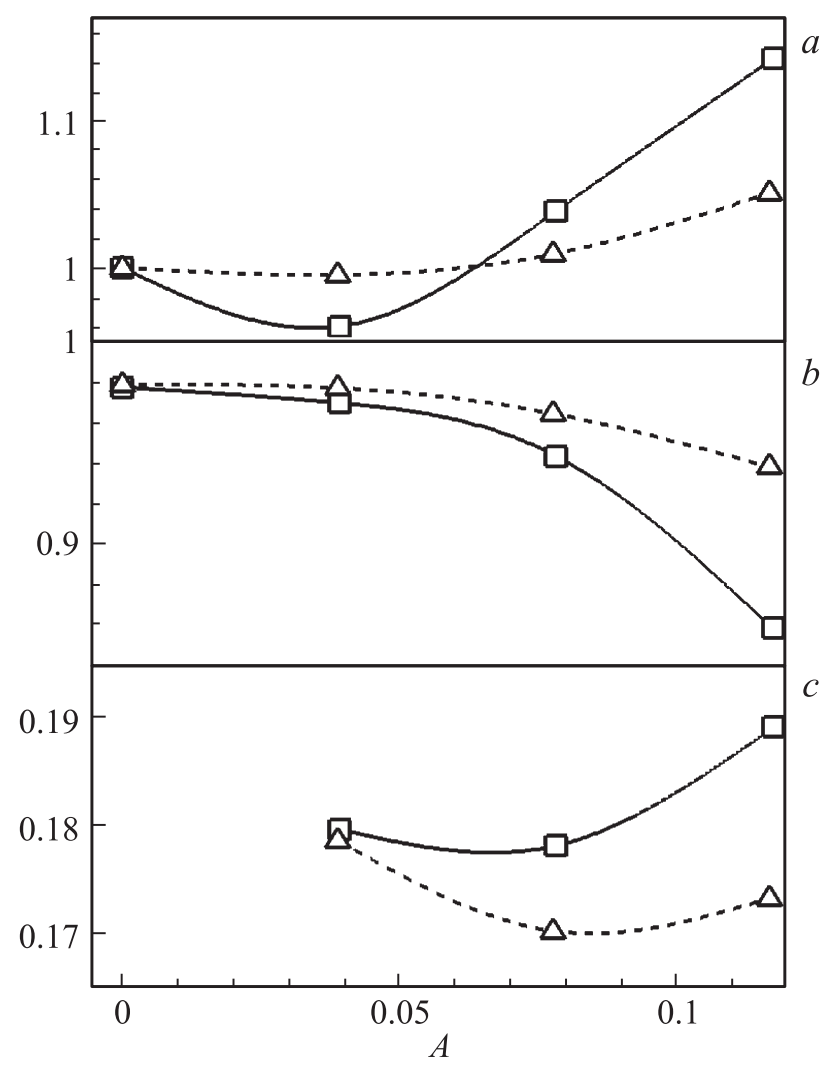

Рис. 2. $a-$ величины $\mathrm{rms} u_{\varphi} / \mathrm{rms} u_{\varphi}(A=0) ; b-$ величины $\operatorname{rms}\left(u_{\varphi}-\right.$ $\left.-u_{c o h}\right) / \mathrm{rms} u_{\varphi} ; c-$ величины $\operatorname{rms}(\Delta \chi) / \Omega_{10}$. Сплошные линии и квадратные символы - $f=0.01 \mathrm{~Hz}$, прерывистые линии и треугольные символы $f=0.08 \mathrm{~Hz}$.

Сигнал $u_{\varphi}$ можно представить в виде суммы трех составляющих средней, когерентной и случайной, такое разложение использовалось, например, в [4]. В присутствии модуляции свойства турбулентности могут изменяться во времени $[8,9]$. Поэтому при вычислении когерентной составляющей в настоящей работе временной сигнал представлялся в виде суммы внутренних мод [12], каждая из которых характеризуется

Письма в ЖТФ, 2017, том 43, вып. 10 
медленно меняющейся во времени частотой, и малого остатка. Мода такого разложения на частоте модуляции использовалась в настоящей работе в качестве когерентной составляющей и представляла собой модулированный по амплитуде сигнал. На рис. 2, $b$ показаны зависимости от амплитуды модуляции среднеквадратичных отклонений пульсаций $u_{\varphi}$ с вычетом когерентной составляющей $u_{c o h}$, нормированные на ту же величину без вычета когерентной составляющей $-\operatorname{rms}\left(u_{\varphi}-u_{c o h}\right) / \mathrm{rms} u_{\varphi}$. Во всем диапазоне амплитуд модуляции наблюдается монотонное снижение этой величины, увеличивающееся с уменьшением частоты модуляции.

Модуляция скорости вращения может рассматриваться как внешняя по отношению к течению вынуждающая сила, вызывающая синхронизацию течения. Оценить уровень синхронизации можно по поведению мгновенных величин фазы и частоты сигнала [9]. Значение мгновенной фазы $\Psi(t)$ сигнала скорости $x(t)$ определяется как $\Psi(t)=\operatorname{arctg}(y(t) / x(t)) ; y(t)$ - ортогональное дополнение к $x(t)$, которое вычисляется как преобразование Гильберта ряда $x(t)$ [12]. Тогда $\chi(t)=\partial(\Psi(t)) / \partial t-$ мгновенная частота. Рассмотрим среднеквадратичные отклонения разности между мгновенными частотами, соответствующими скорости вращения внутренней сферы и скорости в выбранной точке течения, нормированные на величину средней угловой скорости: $\operatorname{rms}(\Delta \chi) / \Omega_{10}$ (рис. 2,c). Так же как и для турбулентных пульсаций скорости (рис. 2,a), в зависимости разности мгновенных частот от величины $A$ наблюдается локальный минимум, который соответствует наиболее высокому уровню синхронизации. Чем ниже $f$, тем меньше величина $A$, соответствующая локальному минимуму. Отметим, что локальный минимум при $f=0.08 \mathrm{~Hz}$ соответствует диапазону амплитуд, в котором происходит замена спектров, характерных для трехмерной турбулентности, на спектры, характерные для двумерной турбулентности (см. таблицу). Это означает, что в случае двумерной турбулентности уровень синхронизации выше, чем в случае трехмерной турбулентности.

Таким образом, модуляция скорости вращения может приводить к снижению уровня турбулентных пульсаций скорости, и этот уровень в случае двумерной турбулентности ниже, чем в случае трехмерной. Переход от трехмерной турбулентности к двумерной под действием модуляции возможен в случае высокого уровня синхронизации.

Письма в ЖТФ, 2017, том 43, вып. 10 
Работа выполнена при финансовой поддержке РФФИ, проект № 16-05-00004.

\section{Список литературы}

[1] Монин А.С., Яглом А.М. Статистическая гидромеханика. Т. 2. М.: Наука, 1967.

[2] Данилов С., Гурарий Д. // УФН. 2000. Т. 170. № 9. С. 921-968.

[3] Boffetta G., Ecke R. // Ann. Rev. Fluid Mech. 2012. V. 44. P. 427-451.

[4] Kit E. et al. // J. Fluid Mech. 2007. V. 589. P. 479-507.

[5] Hrebtov M., Ilyushin B., Krasinsky D. // Phys. Rev. E. 2010. V. 81. P. 016315.

[6] Farazmand M., Kevlahan N., Protas B. // J. Fluid Mech. 2011. V. 668. P. 202 222.

[7] Smith L. // Phys. Fluids. 1999. V. 11. N 6. P. 1608-1622.

[8] Жиленко Д.Ю., Кривоносова О.Э. // Письма в ЖТФ. 2013. Т. 39. В. 1. С. 62 70.

[9] Жиленко Д.Ю., Кривоносова О.Э. // Письма в ЖЭТФ. 2015. Т. 101. В. 8. C. $583-588$

[10] Меламед Л.Э. // Письма в ЖТФ. 2015. Т. 41. В. 24. С. 23-28.

[11] Shang X.-D., Xia K.-Q. // Phys. Rev. E. 2001. V. 64. P. 065301.

[12] Huang N.E., Shen Z., Long S.R. // Ann. Rev. Fluid Mech. 1999. V. 31. P. $417-$ 457. 